\title{
Erratum: Earth's spheroidal motion induced by a gravitational wave in flat spacetime [Phys. Rev. D 100, 044048 (2019)]
}

\author{
Josipa Majstorović $\odot$, Séverine Rosat, and Yves Rogister
}

(Received 21 December 2020; published 21 January 2021)

DOI: 10.1103/PhysRevD.103.029901

In the paper, "Earth's spheroidal motion induced by a gravitational wave in flat space-time" by Majstorović et al. [1], we revisited the theoretical model describing the interaction between an astrophysical gravitational wave (GW) with Earth's normal modes in the millihertz frequency band based on Ben-Menahem's work (1983) [2]. The revisited model provides us with new estimates of the radial and horizontal displacements represented by the relation $\langle 55\rangle$ (in the following, the equations from [1] are inserted in angle brackets). These values are calculated using the general relations $\langle 38\rangle$ and $\langle 39\rangle$ by introducing several assumptions that lead to relations $\langle 52\rangle$ and $\langle 53\rangle$. Those assumptions include: first, we consider displacement for ${ }_{0} \mathrm{~S}_{2}$; second, the source is monochromatic and at the resonance with the normal mode's eigenfrequency; third, the sensor position is at the equator and the source angles are $\left\{e=\frac{\pi}{2}, \lambda=0, \nu=0\right\}$; fourth, we introduce the corrections for the perturbation in the gravitational potential and free-air changes in gravity. In this Erratum, we are acknowledging that a lapse has been made in the model interpretation when calculating the displacement values $\langle 55\rangle$. To obtain the aforementioned values, we considered the time instance $t=0$, thus leaving only the terms associated with the cosine function in $\langle 52\rangle$ and $\langle 53\rangle$. However, the resonance cannot be established at $t=0$, and instead of the cosine function, we should consider the terms next to the sine function. That leads us to the configuration where the radial displacement is dominant and Q dependent as it should be for any resonant system; see, e.g., the equations for a resonance bar [3]; thus, we have

$$
\begin{aligned}
{ }_{0} s_{2, r} & \approx-\frac{1}{4 \pi} h_{0} \alpha_{0} \alpha_{2} \bar{U}_{2} \frac{{ }_{0} \omega_{2}}{{ }_{0} \gamma_{2}\left({ }_{0} \omega_{2}{ }^{2}+{ }_{0} \gamma_{2}{ }^{2}\right)} \\
& \approx-\frac{1}{2 \pi} h_{0} \alpha_{0} \alpha_{20} \bar{U}_{2} \frac{Q}{{ }_{0} \omega_{2}{ }^{2}} \frac{4 Q^{2}}{4 Q^{2}+1}, \\
{ }_{0} s_{2, \theta} & \approx-\frac{1}{2 \pi \sqrt{6}} h_{0} \alpha_{0} \alpha_{2} \bar{V}_{2} \frac{{ }_{0} \gamma_{2}}{{ }_{0} \gamma_{2}\left({ }_{0} \omega_{2}{ }^{2}+{ }_{0} \gamma_{2}{ }^{2}\right)} \\
& \approx-\frac{1}{2 \pi \sqrt{6}} h_{0} \alpha_{0} \alpha_{20} \bar{V}_{2} \frac{1}{{ }_{0} \omega_{2}{ }^{2}} \frac{4 Q^{2}}{4 Q^{2}+1} .
\end{aligned}
$$

Please note that all the figures in Majstorovic et al. [1] were plotted using the full expressions $\langle 52\rangle$ and $\langle 53\rangle$ and not the erroneous approximation, so they are correct.

New displacement and gravity estimates. - At this point, to obtain the final radial and tangential displacement estimates, one needs to utilize the MINEOS normalized values from the Table II in [1]. Further, the displacements are multiplied with the radius of the Earth $(6371 \mathrm{~km})$ to consider for the displacement normalization. The radial and tangential induced ground displacements are

$$
\begin{aligned}
& { }_{0} s_{2, r} \approx 7.1 \cdot 10^{7} h_{0} \mathrm{~m}, \\
& { }_{0} s_{2, \theta} \approx 2.5 \cdot 10^{4} h_{0} \mathrm{~m} .
\end{aligned}
$$

We can calculate the acceleration values by multiplying (3) with ${ }_{0} \omega_{2}{ }^{2}$,

$$
\begin{aligned}
& { }_{0} a_{2, r} \approx 2.7 \cdot 10^{11} h_{0} \mathrm{~nm} / \mathrm{s}^{2}, \\
& { }_{0} a_{2, \theta} \approx 9.4 \cdot 10^{7} h_{0} \mathrm{~nm} / \mathrm{s}^{2} .
\end{aligned}
$$


If we set $h_{0}=10^{-21}$, we arrive at the new displacement values,

$$
\begin{aligned}
& { }_{0} s_{2, r} \approx 7.1 \times 10^{-5} \mathrm{~nm}, \\
& { }_{0} s_{2, \theta} \approx 2.5 \times 10^{-8} \mathrm{~nm},
\end{aligned}
$$

and gravity values

$$
\begin{aligned}
& { }_{0} a_{2, r} \approx 2.7 \times 10^{-10} \mathrm{~nm} / \mathrm{s}^{2}, \\
& { }_{0} a_{2, \theta} \approx 9.4 \times 10^{-14} \mathrm{~nm} / \mathrm{s}^{2} .
\end{aligned}
$$

On the MINEOS normalization.-Next, we elaborate the use of the Earth's radius as a correct displacement normalization, applied in Majstorovic et al. [1], by exploring the relation between the normalization introduced in Dahlen and Tromp (1998) [4] (further on DH98) and in the MINEOS software package [5,6]. Considering only those physical quantities that have units in (1), we have (dropping subscripts $l$ and $m$ )

$$
s_{r}=\alpha \bar{U} \frac{1}{\omega^{2}}=\left(\beta^{2} \rho a^{2}\right) \bar{U} \frac{1}{\omega^{2}},
$$

thus performing the dimensional analysis of the relation (7), and by adopting [L] for length, $[\mathrm{M}]$ for mass, and $[\mathrm{T}]$ for time, we obtain:

$$
\operatorname{dim} s_{r}=\frac{[\mathrm{L}]^{2}}{[\mathrm{~T}]^{2}} \frac{[\mathrm{M}]}{[\mathrm{L}]^{3}}[\mathrm{~L}]^{2} \frac{1}{[\mathrm{M}]}[\mathrm{T}]^{2}=[\mathrm{L}]
$$

Several assumptions are used in (7) and (8): First, $\alpha \approx \mu(a) a^{2}$ based on relation $\langle 36\rangle$ in [1]; second, we use the definition for the shear modulus $\mu=\beta^{2} \rho$, where $\beta$ is the shear-wave velocity; third, for the eigenfunction unit, we use $\frac{1}{[\mathrm{M}]}$ as in DH98. When calculating the actual displacement values, we use the eigenfunctions computed by MINEOS; thus, we need to be sure that the normalization is correctly suppressed later on. From the MINEOS, the normalization of the eigenfunction is $\left[\frac{\mathrm{T}^{2}}{\mathrm{M}}\right]$. Hence, if we note $s_{r}$ as the displacement in DH98 and $s_{r, n}$ as the displacement in MINEOS, the relation between the two is established as

$$
s_{r, n} \approx \frac{s_{r}}{N} \approx\left(\frac{\beta}{v_{n}}\right)^{2} \frac{\rho}{\rho_{n}}\left(\frac{a}{r_{n}}\right)^{2}\left(\frac{U}{U_{n}}\right)^{2} \frac{1}{\left(\frac{\omega}{\omega_{n}}\right)^{2}},
$$

where $v_{n}, \rho_{n}, r_{n}, U_{n}, \omega_{n}$ are MINEOS normalization for velocity, density, radius, eigenfunction, and eigenfrequency, respectively, listed in Tab I. To obtain displacement values as in DH98, we need to multiply $s_{r, n}$, with

$$
N=v_{n}^{2} \cdot \rho_{n} \cdot r_{n}^{2} \cdot U_{n}^{2} \cdot \frac{1}{\omega_{n}^{2}}=r_{n}\left(\pi G \rho_{n}\right),
$$

which gives

$$
s_{r}=s_{r, n} N=s_{r, n} r_{n}\left(\pi G \rho_{n}\right),
$$

which has a unit $\left[\frac{\mathrm{L}}{\mathrm{T}^{2}}\right]$. Since we need to cancel the time unit, we further multiply our displacement with the frequency normalization that has a time unit [T] (Tab. I) and is defined as

$$
\omega_{n}=\frac{v_{n}}{r_{n}}=\frac{1}{\sqrt{\pi G \rho_{n}}} .
$$

Combining (11) and (12), we obtain:

$$
s_{r}=s_{r, n} r_{n}\left(\pi G \rho_{n}\right) \omega_{n}^{2}=s_{r, n} r_{n}\left(\pi G \rho_{n}\right) \frac{1}{\pi G \rho_{n}}=s_{r, n} r_{n},
$$


TABLE I. MINEOS normalization forms for the eigenfunctions $W(r), U(r), V(r)$, the average density $\rho_{n}$, the radius of the Earth $r_{n}$, the velocity $v_{n}$, and the frequency $\omega_{n}$ from [6], where $G=6.67408 \times 10^{-11} \mathrm{~m}^{3} \mathrm{~kg}^{-1} \mathrm{~s}^{-2}$ is the gravitational constant.

\begin{tabular}{ll}
\hline \hline Normalization & \multicolumn{1}{c}{ Definition } \\
\hline Torodial eigenfunctions & $\omega^{2} \int_{0}^{r_{n}} \rho(r) W^{2}(r) r^{2} d r=1$ \\
Spheroidal eigenfunctions & $\omega^{2} \int_{0}^{r_{n}} \rho(r)\left[U^{2}(r)+V^{2}(r)\right] r^{2} d r=1$ \\
Density & $\rho_{n}=5515\left[\frac{\mathrm{kg}}{\mathrm{m}^{3}}\right]$ \\
Radius & $r_{n}=6371000[\mathrm{~m}]$ \\
Velocity & $v_{n}=\frac{r_{n}}{\sqrt{\pi G \rho_{n}}}[\mathrm{~ms}]$ \\
Frequency & $\omega_{n}=\frac{v_{n}}{r_{n}}[\mathrm{~s}]$ \\
\hline
\end{tabular}

which has a unit of [L]. Finally, canceling MINEOS normalization is achieved only by multiplying the displacement $s_{r, n}$ with the radius of the Earth.

New detection threshold. - With the new acceleration estimates (6), we recalculate the signal detection threshold in Earth by assuming that the average environmental seismic noise level is defined by the new low noise model (NLNM) [7] (see [1]). Here, the NLNM model is considered as a benchmark that, however, is surpassed by the sensitivity of the superconducting gravimeters (SGs) below $1 \mathrm{mHz}$, where one would search for the gravitationally induced signal. For comparison, the average and the minimum reached sensitivity by SGs are approximately $-165 \mathrm{~dB}$ at $1 \mathrm{mHz}$ and $-175 \mathrm{~dB}$ at $1 \mathrm{mHz}$ (Black Forest Observatory, Germany), respectively [8,9]. To answer how large the monochromatic signal needs to be to be detectable when embedded in the noise, we need to obtain a rough estimate of the noise standard deviation that represents the seismic background noise. This value is obtained from the PSD defined with NLNM. The PSD is frequency dependent, and to define the noise standard deviation from the PSD, we can use the definition,

$$
\sigma^{2}=\int_{f-\Delta f / 2}^{f+\Delta f / 2} P(f) d f
$$

where $\sigma^{2}$ is the variance of the signal in the frequency band $\Delta f$, and $P(f)$ is the frequency dependent PSD. Thus, if we integrate the PSD over the frequency band of interest, we can obtain the variance of the signal in that band. Therefore, if the PSD is constant near the frequency $f$, we obtain,

$$
\sigma^{2}=P(f) \Delta f
$$

the variance of the signal in question depends on the frequency bandwidth. There is no exact answer to what is the bandwidth of the target signal. In the case of the ${ }_{0} \mathrm{~S}_{2}$ mode, we can either take the splitting width, which is $\Delta f=20 \mu \mathrm{Hz}$, or the width of the individual singlet broaden due to its Q factor, which would be $\Delta f=5 \mu \mathrm{Hz}$. Since the NLNM is flat in the $\mathrm{mHz}$ band around ${ }_{0} \mathrm{~S}_{2}$ frequency, we set $P(f)=-151.88 \mathrm{~dB}$, and this gives us

$$
\sigma_{\mathrm{NLNM}}=\sqrt{10^{-\frac{151.88}{10}}} \sqrt{\Delta f}
$$

Considering (16), we can calculate the noise standard deviations when the frequency bandwidth is defined for either the entire multiplet or one singlet, and those values are $1.1390 \times 10^{-10} \mathrm{~m} / \mathrm{s}^{2}$ and $5.6949 \times 10^{-11} \mathrm{~m} / \mathrm{s}^{2}$, respectively. From the discussion in [1], we note that signal amplitude needs to satisfy the relation $A>\frac{2 \sigma}{\sqrt{N}}$ to be visible in the noisy data. Thus, to detect the radial component (6) buried in the noisy time series with standard deviations defined earlier, one would need either $L=N \Delta t>\left(\frac{2 \sigma_{\mathrm{NLNM}}}{{ }_{0} a_{2, r}}\right)^{2} \Delta t>4.2 \times 10^{19}$ (multiplet bandwidth) or $2.7 \times 10^{18}$ (singlet bandwidth) years of data with $\Delta t=60 \mathrm{~s}$. One can look at this differently and say that for this particular noise standard deviation and 10 years of recorded data and $\Delta t=60 \mathrm{~s}$ (thus, $N=5256000$ ), one would need the GW amplitude signal to be larger than either $h_{0}>\frac{2 \sigma_{\mathrm{NLNM}}}{a_{0} \sqrt{N}}>$ $3.7 \times 10^{-16}$ (multiplet bandwidth) or $h_{0} \approx 1.8 \times 10^{-16}$ (singlet bandwidth) to be detected on Earth, where ${ }_{0} a_{2, r}=a_{0} h_{0}=270 h_{0} \mathrm{~m} / \mathrm{s}^{2}$.

Conclusion.-We have revised the estimated values of the ground displacement and gravity effects induced by a monochromatic GW in resonance with the ${ }_{0} \mathrm{~S}_{2}$ seismic mode around $54 \mathrm{~min}$. The new radial displacement and surface 
gravity change values are by 3 orders of magnitude larger than the ones provided in [1], thus reaching $7.1 \times 10^{-5} \mathrm{~nm}$ and $2.7 \times 10^{-10} \mathrm{~nm} / \mathrm{s}^{2}$, respectively.

We acknowledge J. Harms for pointing out that our firstly published estimates were underestimated with respect to previous works and helping us to find out our mistake. We are grateful to an anonymous reviewer for helpful comment.

[1] J. Majstorović, S. Rosat, and Y. Rogister, Earth's spheroidal motion induced by a gravitational wave in flat spacetime, Phys. Rev. D 100, 044048 (2019).

[2] A. Ben-Menahem, Excitation of the Earth's eigenvibrations by gravitational radiation from astrophysical sources, II Nuovo Cimento C 6, 49 (1983).

[3] J. Weber, Detection and generation of gravitational waves, Phys. Rev. 117, 306 (1960).

[4] F. A. Dahlen and J. Tromp, in Theoretical Global Seismology (Princeton University Press, Princeton, NJ, 1998), p. 1025.

[5] J. H. Woodhouse, in Seismological Algorithms, Computational Methods and Computer Programs, edited by D. J. Doornbos (Academic Press, London, 1988), Chap. The calculation of eigenfrequencies and eigenfunctions of the free oscillations of the Earth and the Sun, pp. 321-370.

[6] B. M. G. Masters and S. Kientz, Computational infrastructure for geodynamics v. 1.0.2. (2014), https://geodynamics.org/cig/ software/mineos/.

[7] J. R. Peterson, Observations and modeling of seismic background noise, Open-File Report No. 93332. U.S. Department of Interior, Geological Survey, Albuquerque, New Mexico, 1993, https://doi.org/10.3133/ofr93322.

[8] S. Rosat and J. Hinderer, Noise levels of superconducting gravimeters: updated comparison and time stability, Bull. Seismol. Soc. Am. 101, 1233 (2011).

[9] S. Rosat and J. Hinderer, Limits of detection of gravimetric signals on Earth, Sci. Rep. 8, 15324 (2018). 